\title{
Estrategia literaria para la aceptación de la imagen corporal en jóvenes universitarios ecuatorianos
}

\section{Literary strategy for the acceptance of body image in young Ecuadorian university students}

\author{
Álvaro Jiménez-Sáncheza , Jocelyn Patricia Andino-Reinoa , Eliza Carolina Vayas-Ruiza \\ a Departamento de Investigación de la Facultad de Jurisprudencia y Ciencias Sociales, Universidad Técnica de Ambato, Ecuador
}

\begin{abstract}
Resumen
Introducción: El rechazo de nuestra propia imagen corporal puede conducir a problemas de salud graves como la anorexia o la bulimia nerviosa, especialmente entre la población joven. Objetivo: Aplicar una estrategia en edu-entretenimiento para mejorar la imagen corporal de los jóvenes en el centro de la sierra ecuatoriana. Metodología: Se entregó un cuestionario a 788 estudiantes universitarios sobre el grado de aceptación de la imagen corporal (IMC real frente al IMC ideal). La mitad de ellos participó previamente en una estrategia literaria dirigida a escribir un relato corto en parejas relacionado con la anorexia o la bulimia nerviosa. Resultados: El grupo control tiene peor aceptación de su imagen corporal que el grupo experimental. Los participantes valoran la dinámica literaria como bastante entretenida y educativa. No existe relación entre el grado de aceptación de la imagen corporal y la identificación con la historia, pero sí entre esta aceptación y el número de personas cercanas con trastornos alimenticios de este tipo, así como con la valoración de su propia dieta y con sentirse a gusto con su cuerpo. Conclusión: La dinámica utilizada puede ser útil como parte de intervenciones en comunicación sobre salud para tratar de solventar estos problemas.
\end{abstract}

Palabras clave: Jóvenes; imagen corporal; comunicación en salud; literatura; desórdenes alimenticios.

\begin{abstract}
Introduction: The rejection of our own body image can lead to serious health problems such as anorexia or bulimia nervosa, especially among young population. Objective: To apply an edu-entertainment strategy to improve the body image of young people from the center of the Ecuadorian highlands. Methodology: A questionnaire about the degree of acceptance of body image (real BMI vs ideal BMI) was handed out to 788 university students. Half of them previously took part in a literary strategy consisting of writing in pairs a short story related to anorexia or bulimia nervosa. Results: The control group has a worse physical image of themselves compared to the experimental group. Participants evaluated the literary dynamics as quite entertaining and educational. There is no relation between the degree of acceptance of body image and identification with the story, but there is a relation between this acceptance and the number of close people with this type of eating disorders, as well as the assessment of their own diet and feeling comfortable with their own body. Conclusion: The dynamics used can be useful as part of health communication interventions in order to solve these problems.
\end{abstract}

Keywords: Young; body image; communication in health; literature; eating disorders. 


\section{Introducción}

$\mathrm{L}$ a dieta es muy importante para el desarrollo de una vida saludable. Los malos hábitos alimenticios pueden provocar serios problemas de salud. Actualmente, hay muchos países en donde las principales causas de mortalidad de sus ciudadanos se derivan de una dieta incorrecta. Aunque esta problemática ocurre mayormente entre personas mayores, los jóvenes son una población de interés en este campo porque se encuentran en una fase especial en términos de desarrollo evolutivo (Andino-Reino, Jiménez-Sánchez y Vayas-Ruiz, 2019a).

Estos hábitos alimenticios son de vital importancia en esta etapa. Por un lado, está la ingesta de alimentos, que es esencial para el buen crecimiento y desarrollo corporal que caracteriza a estas edades. Por otro lado, la integración de estos cambios físicos, que pueden desencadenar serios problemas si la persona no acepta o evalúa inadecuadamente su imagen corporal.

Según el Instituto Nacional de Estadística y Censo (INEC), en su Encuesta Nacional de Salud y Nutrición (ENSANUT-INEC, 2014), aproximadamente el $26 \%$ de la población ecuatoriana entre 12 y 19 años tiene sobrepeso u obesidad. Con respecto a los trastornos alimenticios (TA) como la anorexia o la bulimia nerviosa, no hay cifras generales al respecto, ya que no se consideran problemas de salud pública (El Comercio, 24 de noviembre, 2014). Sin embargo, algunas investigaciones locales más recientes hablan de una alta incidencia de estos trastornos en la población, especialmente entre mujeres jóvenes (Andino-Reino, Jiménez-Sánchez y Vayas-Ruiz, 2019b; Jiménez-Sánchez et al., 2018). Estos trastornos están influidos por numerosos factores psicológicos, socioculturales y especialmente, por los medios de comunicación y las redes sociales (GonzálezDíaz, 2016; Murga-Eizagaechevarría, 2016).

Cabe recordar que los TA son enfermedades graves pero curables si la persona realiza un tratamiento con psicólogos y médicos especializados. Los TA se caracterizan por un comportamiento patológico contra esta ingesta y una obsesión con el control del peso. De origen multifactorial (biológico, psicológico, familiar y sociocultural), son enfermedades que tienen consecuencias negativas para la salud física y mental de la persona (ACAB, 2017).

Debido a esto, esta investigación tiene como objetivo recurrir a estrategias en edu-entretenimiento como otro elemento más en la lucha para aliviar este tipo de trastornos.

Según la Organización Mundial de la Salud (OMS), la comunicación en salud abarca el estudio y el uso de estrategias de comunicación para informar e influir en las decisiones individuales y comunitarias que mejoran la salud (Mosquera, 2003, citado por RodríguezRoura, Cabrera-Reyes y Calero-Yera, 2018). Aun así, este autor cree que es necesario tener en cuenta los determinantes que pueden cambiar en relación con otros contextos, como las creencias que las personas tienen sobre la adopción de algún tipo de comportamiento 0 los obstáculos y ventajas del entorno social y natural. Estas consideraciones mostrarían la importancia de apreciar la diversidad teórica en el desarrollo de una comunicación en salud efectiva (Jiménez-Sánchez y Vayas-Ruiz, 2018a).

La comunicación en salud abarca múltiples actividades destinadas a reducir los TA, que hacen posible su uso en contextos más locales. Por su parte, la edu-comunicación se utilizaría para diseñar la búsqueda de interacciones colectivas y dialógicas, que dependen del uso de procesos y herramientas de comunicación para garantizar el progreso y el desarrollo humano (De Oliveira, 2009). Es, en resumen, una forma de pedagogía crítica que concibe los procesos educativos, la comunicación, los medios y las tecnologías como herramientas de análisis y acción para la comprensión y transformación del mundo (Barbas, 2012).

La dinámica utilizada en este estudio se basa en la redacción de textos relacionados con los TA. La idea se basa en investigaciones similares en las que el proceso de escritura sobre un tema puede ayudar a crear conciencia sobre ciertos aspectos que se van a tratar (Acosta-Pérez, Jiménez-Sánchez y Paredes-Ruiz, 2019; Jiménez-Sánchez y Vayas-Ruiz, 2018b). Como proponen los autores citados, la dinámica debe estar organizada y bien planificada. Se recomienda una hoja metodológica con la descripción de cada actividad para guiar a cualquier persona interesada en llevarlas a cabo. Por lo general, se componen de aspectos como la duración estimada, la descripción, los objetivos, el desarrollo, los materiales necesarios, la preparación, las sugerencias, la evaluación o los comentarios posteriores a la sesión.

La diferencia entre la dinámica de la lectura y la escritura se aprecia en que en la primera el instructor usa las historias como punto de partida, mientras que en la última los temas son el punto de partida que se discutirá más adelante para crear nuevas historias (Acosta-Pérez, 
Jiménez-Sánchez y Paredes-Ruiz, 2019). De esta manera, la creación de "ficción" puede conducir a un proceso de catarsis en el que el autor refleja su vida diaria en las escenas, tramas y personajes. Esto ofrece una perspectiva más activa y personal que cuando se lee la historia. A su vez, se podría conseguir relacionar las emociones y los sentimientos del autor expresados en el cuento (Cantero, 2005). Sin embargo, en el caso de la escritura, el papel de los alumnos es más activo porque son los autores (Acosta-Pérez, JiménezSánchez y Paredes-Ruiz, 2019; Jiménez-Sánchez y Vayas-Ruiz, 2018b).

La mayoría de las propuestas metodológicas incluyen una parte de cuestiones o conceptos para trabajar en cada actividad. De esta manera, la necesidad de la fase de debate para completar el divertido proceso de aprendizaje educativo es notable. Para abordar la educación basada en valores, se deben evitar los discursos y las explicaciones en las que los roles del alumno se limiten a escuchar 0 responder preguntas sobre el tema presentado (Jiménez-Sánchez y VayasRuiz, 2018a). Es necesario que los alumnos analicen, piensen, critiquen y pregunten, resuelvan sus dudas y mejoren así su capacidad de análisis crítico (Consejería de Salud y Servicios Sanitarios, 2009).

Aunque las guías de actividades sobre este tema son extensas, los estudios cuantitativos sobre su efectividad son bastante escasos. Es por eso que este artículo utiliza una perspectiva más experimental y analítica respecto a una dinámica centrada en el beneficio de la escritura de relatos cortos. Por lo tanto, los objetivos finales de esta investigación son comprobar si la intervención cambia la forma en la que los estudiantes ven los TA; saber cómo influirán las historias cortas para cambiar sus actitudes y conocimientos sobre el tema; y conocer especialmente si la dinámica empleada sirve para mejorar la aceptación de la imagen corporal.

\section{Metodología}

Se recurrió a un diseño de tipo cuasi-experimental, con una primera fase de diagnóstico o exploratoria y seguida de una segunda etapa de intervención pero aplicada a distintos sujetos. En la primera fase se suministró un cuestionario a 397 estudiantes de entre 17 y 30 años pertenecientes a diferentes carreras de la Universidad Técnica de Ambato, en el que se preguntaba acerca del peso y la altura (Jiménez-Sánchez et al., 2018). En la etapa de intervención participaron 391 alumnos (total $=788$ ) y al final de la actividad literaria se les preguntó sobre su peso y altura, entre otras cuestiones relacionadas con la dinámica empleada. La población estudiantil es de 16.000 personas, por lo que se garantizó que la muestra fuese de un mínimo de 376 personas ( $5 \%$ de margen de error y $95 \%$ de nivel de confianza). Las pruebas estadísticas utilizadas fueron la U-Mann Whitney y la correlación de Spearman. Los datos recopilados se analizaron utilizando el paquete estadístico SPSS (versión 23 para Windows 64 bits).

Todos los sujetos participaron de manera voluntaria, sin ánimo de lucro y con el previo consentimiento informado. Se respetó en todo momento el anonimato de los datos y de los participantes, así como el cumplimiento del reglamento dispuesto por el Comité de Ética de la Universidad.

Para implementar la estrategia de comunicación, este estudio utilizó como referencia varios procesos y principios para proyectos en comunicación en salud (Jiménez-Sánchez y Vayas-Ruiz, 2018a), que son: 1. Investigación y análisis; 2. Diseño estratégico; 3. Desarrollo, producción y revisión de materiales y métodos; 4. Gestión, implementación y seguimiento; 5 . Evaluación del impacto; y 6 . Planificación para la continuidad.

Por lo tanto, el primer paso fue obtener el índice de masa corporal (IMC real) de los jóvenes estudiantes y especialmente, el peso y la estatura que les gustaría tener (IMC ideal). Una vez que los resultados fueron investigados y analizados se llevó a cabo la fase de intervención, en la misma universidad, pero esta vez con diferentes estudiantes.

De media, la actividad duró entre 50 y 60 minutos (duración de una lección estándar) e incluyó los siguientes pasos:

- Introducción del instructor y la actividad.

- Organización de los alumnos en parejas.

- Reparto de una hoja con una ilustración donde se presentan las consecuencias de los TA por parejas.

- Breve explicación de los componentes de la historia (introducción, presentación de personajes y contexto, clímax y finalización).

- Escritura del relato corto sobre el tema.

- Facilitación de la guía del instructor durante el proceso de redacción en caso de preguntas o dudas.

- Recopilación de historias y, si hubiera tiempo, lectura de algunas de ellas.

- Aplicación de una nueva versión resumida de los cuestionarios aplicados en la fase exploratoria. 
- Agradecimiento y despedida.

Una vez que se recopilaron los textos, se creó una página web con todos ellos (ver Anexos). Así, el resto de la gente puede leerlos para concienciarse sobre el tema, o bien aprender sobre la dinámica utilizada.

Se solicitó el permiso necesario a los maestros o tutores involucrados, y se concertó una cita para administrar el cuestionario durante el tiempo de clase. En el caso del grupo control que recibió solo el cuestionario, pero no la intervención, el tiempo para esa actividad no superó los 15 minutos.

Para evitar posibles sesgos en las respuestas, no se les informó de que el objetivo del taller era modificar sus actitudes hacia la anorexia y la bulimia nerviosa, solo se les indicó que escribieran relatos cortos que serían publicados para que otras personas los leyeran.

\section{Resultados}

El rango de edad es de 17 a 30 años (media $=21,7$ ). El $36,3 \%$ son hombres y el $62,2 \%$ son mujeres (un $1,5 \%$ no contestaron). Vale la pena mencionar que en el grupo control había menos mujeres (Rho=,107; $p=, 003)$ y jóvenes de más edad (Rho=-,160; $p<, 000$ ).

Los jóvenes que desarrollaron la actividad la definieron con las siguientes palabras: muy bueno, conciencia, creativo, dinámico, divertido, educativo, entretenido, imaginación, informativo, interesante, recreativo y reflexión.

Del uno (mínimo) al diez (máximo), los estudiantes calificaron la actividad como muy educativa (media = $8,1)$ y entretenida (8,1 también). Con la misma escala, respondieron a la pregunta "¿cuánto te has identificado con la historia?", con una media de 3,95. Cuando se les preguntó en el cuestionario acerca de cuántas personas conocían que tuvieran o hubiesen tenido TA, el 17,9\% dijo que no conocía a nadie, el $39 \%$ a muy pocas personas, el $27,7 \%$ a pocas, el $10,3 \%$ ni a muchas ni a pocas, y el $5,1 \%$ a muchas (media $=1,46$ ). Posteriormente, los estudiantes tuvieron que definir dos conceptos, anorexia y bulimia nerviosa, y con sus respuestas mostraron que todos habían aprendido ambos términos.

A la pregunta de por qué hay personas que quieren tener una altura o peso diferente a pesar de estar en buenas condiciones, las expresiones más repetidas fueron: aceptación social, apariencia física, baja autoestima o autoaceptación, por comentarios u opiniones externas, críticas y presiones sociales, cánones de belleza, estereotipos, inconformidad, inseguridad, no sentirse bien, obsesión con el físico y vanidad.

En cuanto a cómo valoran su alimentación (1 mínimo, 10 máximo), la media es de 7,44 , y respecto a si se sienten cómodos con su cuerpo (mismo baremo) la media es de 8,28.

Con respecto a la parte inferencial de la investigación, se comparó la diferencia entre el IMC real y el IMC ideal de los estudiantes que realizaron la actividad frente a los que no. Para averiguar el IMC real se les preguntó sobre el peso y la estatura, y para encontrar el IMC ideal se les preguntó sobre el peso y la estatura que les gustaría tener. Más tarde, en la operacionalización de los datos, las libras se pasaron a kilogramos y la altura de centímetros a metros. Luego, se usó la fórmula para encontrar el IMC: peso (kilogramos) dividido por la altura (metros) al cuadrado. Después se calculó la diferencia entre el IMC real y el IMC ideal, y finalmente se compararon los dos grupos.

Se utilizó la prueba no paramétrica U-Mann Whitney y la correlación de Spearman (Kolmogorov-Smirnov $=p<, 001)$. Los resultados fueron: U-Mann Whitney $=-3,346 ; p<, 001, y$ Rho $=-, 131 ; p<, 001$. Respecto a otras correlaciones significativas (Spearman, $p<, 005$ ), cuanto mayor es la diferencia entre los IMC (peor autoimagen), menos valoran su dieta $(p<, 000)$ y se sienten menos cómodos con su cuerpo $(p<, 000)$, datos también apoyados por la fuerte relación directa entre estas dos últimas variables (Rho $=, 439 ; p<, 000)$.

Por su parte, las mujeres tienen una mayor diferencia entre sus IMC $(p=, 025)$, y también valoran menos su dieta que los hombres $(p=, 020)$. A su vez, dentro del rango de edad investigado, a los más jóvenes les pareció la actividad más educativa $(p=, 002)$ y entretenida $(p=, 030)$, pero también son los que menos valoran su dieta $(p=, 004)$. Además, cuanto mayor es el número de personas conocidas con TA, mayor es la diferencia entre los IMC ( $p=, 002)$ (peor autoimagen), menos valoran su dieta $(p=, 001)$ y se sienten menos a gusto con su cuerpo $(p=, 021)$.

Finalmente, no existe una relación entrelaidentificación con la historia y la mejora de la imagen corporal $(p=, 146)$. Sin embargo, sí que hay una relación positiva entre la identificación con la historia y el número de personas conocidas con TA $(p<, 000)$. Esto también provoca que, a mayor identificación, menos valoren su dieta $(p=, 005)$ y estén menos a gusto con su cuerpo $(p<, 000)$. 


\section{Discusión}

En términos generales, la actividad empleada fue positiva y obtuvo buenas críticas y valoraciones sobre su valor educativo y de entretenimiento. Los participantes apreciaron que les gustara el tema y que les ayudara a aceptarse tal y como son, así como a no pretender parecerse físicamente a nadie. Además, no solo adquirieron conocimiento sobre los trastornos alimenticios, sino que también se dieron cuenta de la importancia de la aceptación de su imagen corporal e incluso pudieron sugerir consejos a las personas con TA. En este sentido, el beneficio de la estrategia utilizada ha sido doble, porque además de recibir información, también han sido capaces de generar una actitud proactiva y a favor de la lucha contra los TA mediante la producción de historias cuyos protagonistas padecían estos desórdenes alimenticios.

A su vez, los consejos dados por los participantes para aquellos que no están satisfechos con su peso o estatura se enfocaron en la aceptación, mejorar la autoestima, amarse a sí mismo, confiar en uno mismo, verse o sentirse bien, ignorar las críticas o los malos comentarios, no dejarse llevar, ser original, acudir a un especialista, valorar y respetarse.

Por su parte, las diferencias significativas entre un grupo y otro demuestran que la disparidad entre el IMC real y el IMC ideal es menor en el grupo que realizó la actividad respecto al grupo control. Es decir, los estudiantes que hicieron la dinámica indicaron un IMC ideal más cercano a su peso y altura real que el grupo control, por lo que se puede confirmar la efectividad de la actividad edu-comunicativa ejecutada.

En cualquier caso, aunque la actividad fue efectiva, todavía había muchos jóvenes (especialmente ellas) que no se sentían cómodos con su cuerpo y a los que les gustaría tener un peso o estatura muy diferente del que realmente tenían, incluso hasta el punto de ser considerado de carácter grave. Por tanto, uno de los grandes desafíos que aún debe abordarse es que el país ecuatoriano trate los TA como problemas de salud pública para que se pueda actuar de manera oficial y, sobre todo, tomar medidas preventivas a nivel institucional trabajando junto con centros de salud, educación y familias (AndinoReino, Jiménez-Sánchez y Vayas-Ruiz, 2019a).

El resultado final delaestrategiade du-entretenimiento no solo afectó a las personas involucradas, sino que también condujo a la creación de un sitio web que contiene todos los textos que escribieron los jóvenes (ver Anexos). El objetivo, como se propuso en el taller, es que el resto del mundo pueda acceder a estos escritos y leerlos o utilizarlos para actividades relacionadas con el proceso de concienciación y educación sobre temas relacionados con los TA.

Además, es interesante la recopilación de propuestas sobre otros temas que les hubiera gustado discutir, como la sexualidad y especialmente el bullying. Con esta demanda sobre otros ámbitos no tan académicos se demuestra la necesidad de una educación informal y el hecho de que no deberían ser enseñados solo por la familia o sus amistades, sino también por instituciones educativas que deberían empoderarse de los problemas que los jóvenes demandan y quieren conocer para el desarrollo de una vida plena y saludable.

Los jóvenes destacaron la importancia de ciertos comentarios despectivos sobre su cuerpo recibidos por sus parientes cercanos y amigos, los cuales podrían influir en una peor aceptación de su imagen corporal. Es por ello que todos (familiares y amigos) y también los medios de comunicación deben ser educados para no promover estándares de belleza que lleven a otros a no sentirse a gusto con su físico, algo que podría derivar en un TA.

Hay que prestar atención a los datos que reflejan cómo conocer a personas con estos trastornos se relaciona con una peor autoimagen, con la valoración de su dieta y con sentirse menos cómodos con su cuerpo. Aunque se pueda pensar primeramente que el hecho de tener casos cercanos con TA hace que sea más difícil para uno mismo caer en estos problemas (por no querer vivir el mismo sufrimiento de esos conocidos), los resultados muestran lo contrario. Esto significa que pesaría más la aceptación social, los comentarios, o la percepción física de las personas que tienen estos trastornos a la hora de influir en los demás para que también estos acaben por desarrollar los problemas mencionados (por querer estar igual de delgadas y "bellas" que estas personas afectadas).

Esto debe plantearse seriamente para futuras intervenciones y para talleres preventivos, porque al detectar posibles casos de TA también habría que observar a las personas cercanas, ya que pueden ser susceptibles de terminar sufriendo estos trastornos (familiares, amigos, etc.). En resumen, los TA no solo afectan a las personas individualmente, sino que también tienen un efecto secundario social muy relevante y a 
tener en cuenta si se quiere prevenir e intervenir (AndinoReino, Jiménez-Sánchez y Vayas-Ruiz, 2019a).

Además, se debe enfatizar en que tampoco hay que vender el discurso de "acepta tu imagen corporal" así como así. El sobrepeso y la obesidad también causan problemas graves y, por lo tanto, es normal que algunas personas quieran tener un peso más bajo y esto no significa que tengan o terminen con un TA. Por lo tanto, siempre es necesario aclarar en estos mensajes y en los talleres que lo ideal es buscar un IMC saludable, y para ello, algunos tendrán que perder o ganar peso corporal.

En definitiva, los TA son un tema pendiente que debe ser tratado desde la adolescencia como parte del aprendizaje extracurricular, por lo que es aconsejable que las instituciones gubernamentales, educativas y familiares colaboren conjuntamente para llevar a cabo estrategias de diversos tipos, adaptadas al contexto sociocultural y, sobre todo, realizadas periódicamente y con objetivos a largo plazo. Aunque las charlas informativas sean la base para que estas personas obtengan conocimiento, también es necesario recurrir a la comunicación en salud y, especialmente, a la educomunicación para reforzar y profundizar en los TA de forma diferente, actualizada y familiar respecto al grupo social en el que se quiere intervenir.

Por último, alguna de las limitaciones a destacar fue, por un lado, que se debería haber medido el peso y la estatura de manera más precisa en vez de preguntar. Sin embargo, al respecto cabe mencionar que no se hizo por falta de tiempo y, además, la diferencia entre el peso y estatura medidos con precisión y el obtenido al preguntar resulta igualmente útil para el objeto de estudio debido a que el error o discrepancia entre el dato real y el supuesto o percibido se vuelve aleatorio y se corrige estadísticamente al haber cientos de participantes.

Por otro lado, otra limitación estaría en el tiempo de ejecución de la actividad, pues tal y como argumentaban lo estudiantes, se quedaba corto. Por ello, se recomienda ampliar no solo la duración en el proceso de escritura, sino también añadir un espacio dedicado a la lectura de algunos texto llamativos y, especialmente, extender la parte final destinada al debate de los TA, pues se considera que el proceso de reflexión derivado es sustancial para el cambio de actitudes y conductas. No obstante, hay que ser consciente de que la posible ampliación de tiempo no es acorde con la duración estándar de muchas de las clases lectivas, lo que ha dificultado que la dinámica pudiera realizarse óptimamente.

\section{Conclusiones}

Se puede concluir que la intervención empleada ha sido efectiva para cambiar la forma en la que los estudiantes ven los TA. La escritura de historias cortas sirve para cambiar las actitudes y conocimientos sobre el tema y a su vez, para mejorar la aceptación de la imagen corporal de uno mismo. Sin embargo, aunque la actividad ha sido positiva, solo debe tomarse como una contribución más dentro de todas las que deben hacerse para abordar los TA.

Las intervenciones específicas como las que se han realizado pueden ser efectivas a corto plazo, pero no tanto a largo plazo, por lo que habría que trabajar longitudinalmente con talleres donde estos participantes tengan un papel activo y no solo pasivo, tal y como ha consistido la actividad literaria propuesta en esta investigación. De esta forma, se podrá crear una sociedad proactiva, responsable, educada y que pueda colaborar en conjunto para mejorar los hábitos saludables del resto de la población.

\section{Contribuciones de los autores}

Los autores participaron igualmente en la elaboración del manuscrito y aprobaron la versión final presentada.

\section{Financiación}

Proyecto de investigación "Estrategias de Eduentretenimiento (Comunicación en Salud) para la promoción de hábitos alimenticios y reducción del consumo alcohólico en los jóvenes ambateños" - (2453CU-P-2017).

\section{Declaración de disponibilidad de datos}

Los datos presentados en este estudio pueden ser solicitados al autor de correspondencia.

Agradecimientos

A todos los estudiantes que participaron en la actividad.

Conflicto de interés

Los autores declaran que no hay conflicto de interés.

\section{Referencias bibliográficas}

ACAB. (2017). ¿Qué son los trastornos de la conducta alimentaria? España: Associació Contra l'Anorèxia i la Bulímia. Recuperado de http://www.acab.org/es/ que-son-los-trastornos-de-la-conducta-alimentaria [fecha de consulta: 20 de octubre de 2020].

Acosta-Pérez, C. P., Jiménez-Sánchez, Á., \& ParedesRuiz, T. (2019). Literary strategy for the reduction of attitudes toward alcohol consumption in 
young ecuadorian university students. In Proceedings of the 13th International Technology, Education and Development Conference (pp. 25302538). Valencia, España: IATED. Recuperado de http://doi.org/10.21125/inted.2019.0691

Andino-Reino, J. P., Jiménez-Sánchez, Á., \& Vayas-Ruiz, E. C. (2019a). Audiovisual Strategy for the acceptance of body image in adolescents from the ecuadorian highlands. In Proceedings of the 13th International Technology, Education and Development Conference (pp. 2558-2563). Valencia, España: IATED. Doi: http://doi.org/10.21125/inted.2019.0695

Andino-Reino, J. P., Jiménez-Sánchez, Á., \& Vayas-Ruiz, E. C. (2019b). Adolescentes y jóvenes: similitudes y diferencias en los hábitos y trastornos alimentarios. Revista Alimentos, Ciencia e Ingeniería, 27(1), 67-79. Recuperado de https://revistas.uta.edu.ec/ erevista/index.php/aci/article/view/928/876

Barbas, Á. (2012). Educomunicación: desarrollo, enfoques y desafíos en un mundo interconectado. Foro de Educación, 10(14), 157-175. Recuperado de http:// www. redalyc.org/articulo. $0 a ?$ id $=447544618012$

Cantero, V. (2005). Apuesta por una concepción psicoafectiva de la práctica didáctica de la literatura en la enseñanza secundaria. Revista de Psicodidáctica, 10(1), 65-74. Recuperado de https:// dialnet.unirioja.es/servlet/articulo?codigo $=1222781$

Consejería de Salud y Servicios Sanitarios. (2009). Ni ogros ni princesas, guía para la educación afectivo-sexual. España: Lena et al. Recuperado de http://www.educandoenigualdad.com/wp-content/ uploads/2014/02/guia_no_ogros_ni_princesas1069. pdf [fecha de consulta: 20 de octubre de 2020].

De Oliveira, D. (2009). Caminos de la educomunicación: utopías, confrontaciones, reconocimientos. Nómadas, (30), 194-207. Recuperado de http:// www.redalyc.org/articulo.oa?id=105112060015

El Comercio. (24 de noviembre, 2014). Los grupos que combaten los trastornos alimenticios. Diario El Comercio. Recuperado de http://www.elcomercio. com/tendencias/grupos-combaten-trastornosalimenticios-crecen.html [fecha de consulta: 20 de octubre de 2020].
ENSANUT-INEC. (2014). Encuesta Nacional de Salud y Nutrición, ENSANUT - ECUADOR 2011 - 2013. Ecuador: Instituto Nacional de Estadística y Censos (INEC). Recuperado de http:// www.ecuadorencifras.gob.ec/documentos/web-inec/ Estadisticas_Sociales/ENSANUT/Presentacion $\% 20$ de $\% 201$ os $\% 20$ principales $\% 20 \% 20$ resultados $\% 20$ ENSANUT.pdf [fecha de consulta: 20 de octubre de 2020].

González-Díaz, E. (2016). Comunicación e información alimentaria vs educación nutricional: ¿Existen concordancias? Revista Española de Comunicación en Salud, S1, S163-S166. Doi: http://dx.doi.org/10.20318/recs.2016.3136

Jiménez-Sánchez, Á., \& Vayas-Ruiz, E. C. (2018a). Video clips to reduce sexist attitudes and justification towards gender violence. Communication \& Society, 31(2), 119-135. 2018. Recuperado de https://www.unav. es/fcom/communication-society/en/articulo.php?art_ id $=676$

Jiménez-Sánchez, Á., \& Vayas-Ruiz, E. C. (2018b). Composition of short stories to reduce sexist attitudes and justification of gender violence. In Proceedings of the 10th International Conference on Education and New Learning Technologies (pp. 10749-10758). Palma, España: IATED. Recuperado de http://doi.org/10.21125/edulearn.2018.2634 [fecha de consulta: 20 de octubre de 2020].

Jiménez-Sánchez, Á., Vayas-Ruiz, E. C., Carrero, Y., Arteaga, C., \& Balarezo, J. (2018). Food habits, anorexia and bulimia, in the youth population of the center of Ecuador. In CICABI 2018, Proceedings of the 1st International Congress of Food Science and Biotechnology - Special issue of Italian Journal of Food Science, 31(5), 75-83. Ambato, Ecuador: Chiriotti Editori. Recuperado de https://doi. org/10.14674/IJFS-1614 [fecha de consulta: 20 de octubre de 2020].

Murga-Eizagaechevarría, N. (2016). e-ciudadanos, esalud y redes sociales. Organizarse y formarse en alimentación y salud. Revista Española de Comunicación en Salud, S1, S163-S166. Doi: http://dx.doi.org/10.20318/recs.2016.3133 
74 // Jiménez-Sánchez, Andino-Reino, \& Vayas-Ruiz

Rodríguez-Roura, S. C., Cabrera-Reyes, L. C., \& Calero-Yera, E. (2018). La comunicación social en salud para la prevención de enfermedades en la comunidad. Humanidades Médicas, 18(2), 384-404. Recuperado de http://scielo.sld. cu/scielo.php?script=sci_arttext\&pid=S1727$81202018000200384 \&$ lng=es\&tlng=es

\section{Anexos}

Web: https://uta2019. wixsite.com/relatos-alimenticios 